\title{
Theoretical bases for determining the velocity and suspended matter concentration in the swirling zone beyond the transverse dam
}

\author{
Masharif Bakiev ${ }^{1 *}$, Kuvonchbek Yakubov ${ }^{1}$, Seytxan Koybakov ${ }^{2}$, Kholmurod Khayitov $^{1}$, and \\ Nodira Bobojanova ${ }^{1}$ \\ ${ }^{1}$ Tashkent Institute of Irrigation and Agricultural Mechanization Engineers, Tashkent, Uzbekistan \\ ${ }^{2}$ Taraz regional university named after M.Kh.Dulaty, Taraz, Kazakhstan
}

\begin{abstract}
Design dependencies to determine the velocity and concentration of suspended matter in the swirling zone beyond the transverse dam in the presence of the initial section of the jet are proposed in the article, using the main provisions of the theory of turbulent jets, the scheme of dividing the flow into hydraulic homogeneous zones: a weakly perturbed core, intense turbulent mixing and reverse currents. The distribution of velocities and concentration of suspended matter (turbidity) in the zone of intense turbulent mixing are affine and obey the theoretical Schlichting-Abramovich relationships; this was substantiated by laboratory and field studies. The equations of continuity and conservation of solid matter along the flow were used to solve the problem. To establish the adequacy of the dependencies obtained, a test problem was implemented in which the velocities in the core and the depth along the flow were assumed constant. The problem was implemented for the following contraction ratios of flow $n_{c}=0,1 ; 0.2 ; 0.3 ; 0.4 ; 0.5$. Tabular and graphic dependencies obtained show that with all contraction ratios of flow, the relative backflow velocities first increase, and at the end of the swirling zone, they sharply decrease. The maximum is observed at the intersection of the outer boundary of the zone of intense turbulent mixing with the protected coast and reaches $\mathrm{m}=0.317$. Comparison of the calculation results with the experimental ones shows their qualitative and quantitative agreement. The relative concentration of suspended matter in reverse currents remains practically constant along the entire length of the swirling zone. It is close to unity for all contraction ratios of flow.
\end{abstract}

\section{Introduction}

The acute shortage of water and land resources in Uzbekistan and other republics of Central Asia raised the question of the development of fertile floodplain lands [1,2]. The formation of solid runoff in the river basin, transportation, and channel processes, sedimentation of suspended matters on the floodplains and deltaic parts of rivers is of great interest to scientists worldwide [3-7]. On the other hand, the population growth in these countries required expanding cities and settlements located close to the coastal parts of the rivers. All

* Corresponding author: bakiev1947@rambler.ru, 
of the above issues could be solved using regulatory structures $[8,9,10]$. The construction of large channel reservoir waterworks facilitates the solution to the problem due to the cutting the river hydrograph.

For the first time in world practice, bilateral regulation of the Amu Darya river bed below the Tuyamuyun hydroelectric complex was realised at a length of $185 \mathrm{~km}$. Transverse dams contracting the river channel were used as regulatory structures $[11,12]$. Methods for calculating the velocity field, the distance between the dams, the marks of the dam's crest and their stability were developed in [13-15]. In the process of interaction with the flow beyond the dams, intensive siltation of the space between the dams occurs [16-18]. The forecast of sedimentation in the inter-dam space remains poorly understood.

\section{Methods}

In the theoretical solution, the basic provisions of the theory of turbulent jets propagating in a limited space $[19,20]$ were used to divide the flow into homogeneous zones: a weakly perturbed core, intense turbulent mixing, and reverse currents. Experimental studies were conducted in a chute with dimensions of $40 \times 60 \times 800 \mathrm{~cm}$, a bottom slope $i=0.0002$, with the following characteristics of the flow and the blind dam: Froude number less than 0.35 , Reynolds number more than 10000 , the water flow rate from 5 to $25 \mathrm{l} / \mathrm{s}$, contraction ratio of flow $\mathrm{n}_{\mathrm{c}}=0.1 ; 0.2 ; 0.3 ; 0.5$, dam erection angle from $30^{\circ}$ to $90^{\circ}$. Water velocity was measured with a micro-spinner (manufactured in the SANIIRI) at five points along the depth.

\section{Results and discussion}

The studies conducted earlier have established the affinity of the velocity field in the zone of intense turbulent mixing (Fig. 1), which obeys the theoretical Schlichting-Abramovich dependence $[19,20]$, and has the following form for the initial section of the jet (Fig. 1)

$$
\frac{U_{\mathrm{g}}-U}{U_{\mathrm{g}}-U_{\mathrm{H}}}=\left(1-\eta^{1,5}\right)^{2}
$$

where $U_{\mathrm{g}}, U, U_{\mathrm{H}}$ are the velocities in the core, in the zone of turbulent mixing and reverse currents;

$\eta=\frac{y_{2}-y}{y_{2}-y}=\frac{y_{2}-y}{b}$; here $y_{2}, y_{1}$ are the outer and inner boundaries of the intensive mixing zone of width $\mathrm{b}$, which at the initial section is $b=0,27 \mathrm{X}$. 


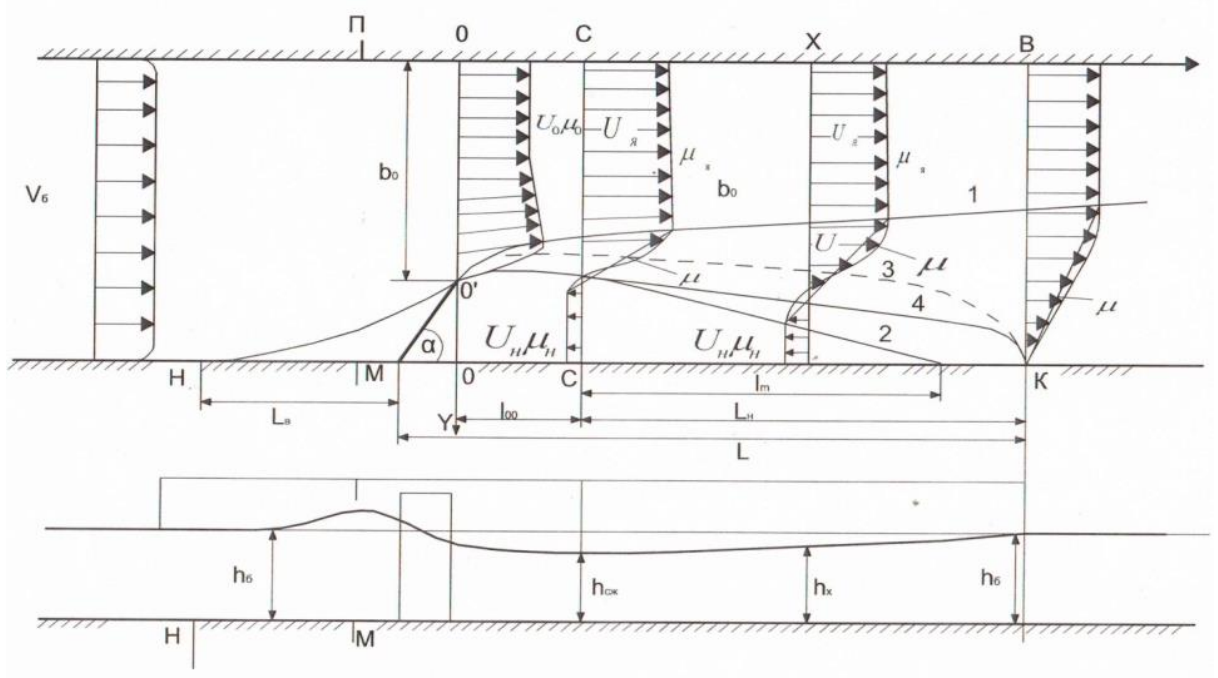

Fig. 1. Design scheme for one-way contraction of the flow by a blind dam.

Since lowland rivers carry a large amount of suspended sediment, as shown by field observations conducted on the Amu Darya River [18], a certain part of the suspended sediment settles in the swirling zone between the dams (Fig. 1)

When solving the problem, the distribution of the suspension concentration in the zone of intense turbulent mixing for the initial section of the jet was taken as (see Fig. 2) [19]:

$$
\frac{\mu_{\mathrm{g}}-\mu}{\mu_{\mathrm{g}}-\mu_{\mathrm{H}}}=(1-\eta)
$$

where $\mu_{\mathrm{g}}, \mu_{\mathrm{H}}, \mu$ are the concentrations of suspended matter in the core, in reverse currents and the zone of turbulent mixing; we assume that $\mu_{\mathrm{g}}=\mu_{0} ; \eta=\frac{\gamma_{2}-\gamma}{b}$.

Field studies in the dam No. 30 [18] confirmed the acceptability of dependence (2)

It is necessary to establish the patterns of changes in the relative velocities in the swirling zone and the relative concentration of suspended matter.

To solve the problem, the equations of continuity and conservation of solid matter along the flow were used for the O-O contraction sections and the X-X section [5]

$$
\begin{gathered}
U_{0} b_{0} h_{0}=U_{\text {я }} b_{\mathrm{g}} h+h \int_{\gamma_{1}}^{\gamma_{2}} U d y+U_{\mathrm{H}} b_{\mathrm{H}} h \\
U_{0} b_{0} h_{0} \mu_{0}=U_{\mathrm{g}} b_{\mathrm{g}} h \mu_{0}+h \int_{\gamma_{1}}^{\gamma_{2}} U \mu d y+U_{\mathrm{H}} b_{\mathrm{H}} h \mu_{\mathrm{H}}
\end{gathered}
$$

We divide equation (3) by $U_{0} b_{0} h_{0}$ and equation (4) by $U_{0} b_{0} h_{0} \mu_{0}$

$$
\begin{gathered}
\frac{h_{0}}{h}=\frac{U_{\mathrm{g}}}{U_{0}} \frac{b_{\mathrm{g}}}{b_{0}}+\int_{\gamma_{1}}^{\gamma_{2}} \frac{U}{U_{0}} \frac{d y}{b_{0}}+\frac{U_{\mathrm{H}}}{U_{0}} \frac{b_{\mathrm{H}}}{b_{0}} \\
\frac{h_{0}}{h}=\frac{U_{\mathrm{g}}}{U_{0}} \frac{b_{\mathrm{g}}}{b_{0}}+\int_{\gamma_{1}}^{\gamma_{2}} \frac{U}{U_{0}} \frac{\mu}{\mu_{0}} \frac{d y}{b_{0}}+\frac{U_{\mathrm{H}}}{U_{0}} \frac{b_{\mathrm{H}}}{b_{0}} \frac{\mu_{\mathrm{H}}}{\mu_{0}}
\end{gathered}
$$

From equations (1) and (2), we obtain

$$
\frac{U}{U_{0}}=1-(1-m)\left(1-\eta^{1,5}\right)^{2}
$$




$$
\frac{\mu}{\mu_{0}}=1-(1-n)(1-\eta)
$$

where $m=\frac{U_{\mathrm{H}}}{U_{0}}$ is the relative velocity of reverse currents;

$n=\frac{\mu}{\mu_{0}}$ is the relative concentration of suspended matter.

Performing the integration in (5) and (6) with (7) and (8), after some transformations, we obtain

$$
\begin{gathered}
\bar{h}_{0}=\bar{U}_{\mathrm{g}} \overline{b_{\mathrm{g}}}+\bar{b}(0,55+0,45 m)+m \overline{b_{\mathrm{H}}} \\
\bar{h}_{0}={\overline{U_{\mathrm{g}}}}_{\overline{\mathrm{g}}}+\bar{b}(-0,52+1,07 n+1.02 m-0,57 m n)+m n \overline{b_{\mathrm{H}}}
\end{gathered}
$$

From (9) $\bar{h}_{0}=\overline{U_{\mathrm{g}}} \overline{b_{\mathrm{g}}}+0,55 \bar{b}+0,45 \bar{b} m+m \overline{b_{\mathrm{H}}}=\overline{U_{\mathrm{g}}} \overline{b_{\mathrm{g}}}+0,55 \bar{b}+m\left(0,45 \bar{b}+\overline{b_{\mathrm{H}}}\right)$ we obtain

$$
m=\frac{\overline{h_{0}}-\overline{U_{\mathrm{g}} \bar{b}_{\mathrm{g}}}-0,55 \bar{b}}{0,45 \bar{b}+\overline{b_{\mathrm{H}}}}
$$

From $(10) \bar{h}_{0}-\overline{U_{\mathrm{g}}} \overline{b_{\mathrm{g}}}+\bar{b}(0,52-1,02 m)=n\left(1,07 \bar{b}-0,57 \bar{b} m+m \overline{b_{\mathrm{H}}}\right)$ we obtain

$$
n=\frac{\overline{h_{0}}-\overline{U_{\mathrm{g}} \bar{b}_{\mathrm{g}}}+\bar{b}(0,52-1,02 m)}{1,07 \overline{b-}\left(0,57 b-b_{\mathrm{H}}\right) m}
$$

To check the adequacy of the theoretical dependencies obtained, a test problem was implemented, where (formulas (11) and (12)) it was assumed that the velocities in the core and the depth remain constant and equal to the velocity and depth at the initial section. In this case, we come to the following dependencies:

The relative backflow velocities are determined as

$$
m=\frac{U_{H}}{U_{0}}=\frac{1-\bar{B}_{g}-0,55 \bar{B}}{0,45 \bar{B}+\bar{\theta}_{H}}
$$

The relative concentration of suspended matter (turbidity) in reverse currents is

$$
n=\frac{1-\bar{B}_{я}+\bar{B}(0,52-1,02 m)}{1,07 \bar{B}-\left(0,57 \bar{B}-\overline{\boldsymbol{B}}_{H}\right) m}
$$

where $b=0,27 x ; \bar{b}=0,27 \zeta ;$ here $\zeta=x / B_{0}$ The core width is $\boldsymbol{s}_{\Omega}=\boldsymbol{B}_{0}-0,11 x$; $\overline{\boldsymbol{B}}_{я}=1-0,11 \zeta$

The calculation is performed for the contraction ratios $n_{c}=0,1 ; 0,2 ; 0,3 ; 0,4 ; 0,5$.

The test problem results are shown in Table 1 for the contraction ratio $n_{c}=0.1$ and in Table 2 - for all considered contraction ratios. 
Table 1. Relative backflow velocities and concentration of suspended matter beyond the blind dam at $n_{c}=0.1$

\begin{tabular}{|c|c|c|c|c|c|c|}
\hline$B$ & $l_{\text {Д }}$ & $\alpha_{\text {Д }}$ & $x$ & $\boldsymbol{B}_{0}$ & $\zeta=x / \boldsymbol{B}_{0}$ & $\bar{B}$ \\
\hline 40 & 4 & $90^{\circ}$ & 5 & 36 & 0.139 & 1.11 \\
\hline 40 & 4 & $90^{\circ}$ & 10 & 36 & 0.278 & 1.11 \\
\hline 40 & 4 & $90^{\circ}$ & 15 & 36 & 0.417 & 1.11 \\
\hline 40 & 4 & $90^{\circ}$ & 20 & 36 & 0.556 & 1.11 \\
\hline 40 & 4 & $90^{\circ}$ & 25 & 36 & 0.694 & 1.11 \\
\hline 40 & 4 & $90^{\circ}$ & 28 & 36 & 0.778 & 1.11 \\
\hline 40 & 4 & $90^{\circ}$ & 36 & 36 & 1.000 & 1.11 \\
\hline
\end{tabular}

Table 1 continued

\begin{tabular}{|c|c|c|c|c|}
\hline$\overline{\boldsymbol{B}}_{\boldsymbol{\gamma}}$ & $\overline{\boldsymbol{B}}$ & $\overline{\boldsymbol{B}}_{\boldsymbol{H}}$ & $m$ & $n$ \\
\hline 0.985 & 0.0375 & 0.0875 & -0.0511 & 0.998 \\
\hline 0.969 & 0.075 & 0.066 & -0.1077 & 0.991 \\
\hline 0.954 & 0.1125 & 0.043 & -0.1707 & 1 \\
\hline 0.939 & 0.15 & 0.021 & -0.2414 & 0.997 \\
\hline 0.924 & 0.1875 & 0.000 & -0.3169 & 1 \\
\hline 0.914 & 0.197 & 0.000 & -0.2555 & 1 \\
\hline 0.890 & 0.221 & 0.000 & -0.1167 & 1 \\
\hline
\end{tabular}

Table 2. Relative backflow velocities along the length of the swirling zone at various contraction ratios $n_{c}$

\begin{tabular}{|c|c|c|c|c|c|}
\hline \multicolumn{2}{|c|}{$\mathrm{n}_{\mathrm{c}}=0.1, \zeta=x / B_{0}$} & \multicolumn{2}{c|}{$\mathrm{n}_{\mathrm{c}}=0.2 ; \zeta=x / B_{0}$} & \multicolumn{2}{c|}{$\mathrm{n}_{\mathrm{c}}=0.3 ; \zeta=x / B_{0}$} \\
\hline & $\mathrm{m}$ & & $\mathrm{m}$ & & $\mathrm{m}$ \\
\hline 0 & 0 & 0 & 0 & 0 & 0 \\
\hline 0.139 & -0.051 & 0.313 & -0.051 & 1.071 & -0.107 \\
\hline 0.278 & -0.108 & 0.625 & -1.07 & 1.429 & -0.147 \\
\hline 0.417 & -0.171 & 0.938 & -0.169 & 1.786 & -0.191 \\
\hline 0.556 & -0.241 & 1.250 & -0.238 & 2.143 & -0.238 \\
\hline 0.694 & -0.317 & 1.563 & -0.317 & 2.679 & -0.317 \\
\hline 0.778 & -0.255 & 1.875 & -0.218 & 3.214 & -0.218 \\
\hline 1.0 & -0.117 & 2.188 & -0.132 & 3.571 & -0.159 \\
\hline
\end{tabular}

Table 2 continued

\begin{tabular}{|c|c|c|c|}
\hline \multicolumn{2}{|c|}{$\mathrm{n}_{\mathrm{c}}=0.4 ; \zeta=x / B_{0}$} & \multicolumn{2}{c|}{$\mathrm{n}_{\mathrm{c}}=0.5 \zeta=x / \mathrm{B}_{0}$} \\
\hline & $\mathrm{m}$ & & $\mathrm{m}$ \\
\hline 0 & 0 & 0 & 0 \\
\hline 1.667 & -0.107 & 2.0 & -0.083 \\
\hline 2.5 & -0.169 & 3.0 & -0.131 \\
\hline 3.333 & -0.238 & 4.0 & -0.182 \\
\hline 3.750 & -.0276 & 5.0 & -0.238 \\
\hline 4.167 & -0.317 & 6.25 & -0.317 \\
\hline 5.208 & -0.195 & 7.0 & -0.255 \\
\hline 5.417 & -0.173 & 8.0 & -0.182 \\
\hline
\end{tabular}




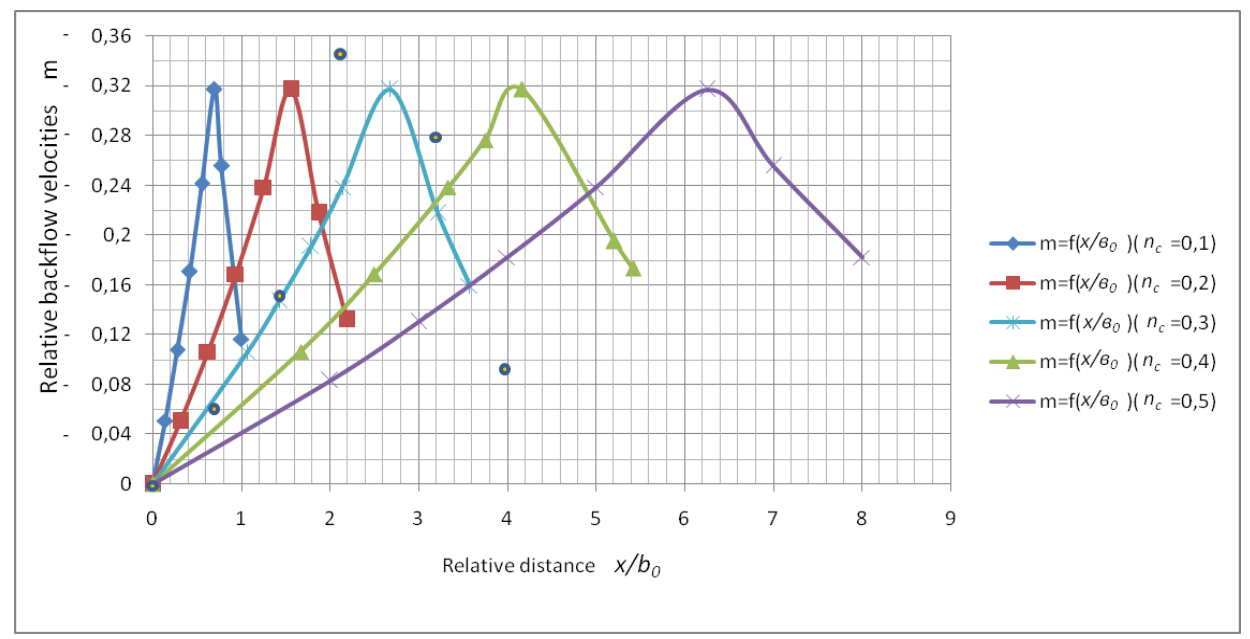

Fig. 2. Graphs of dependence of the relative backflow velocities on the relative length of the swirling zone at various contraction ratios

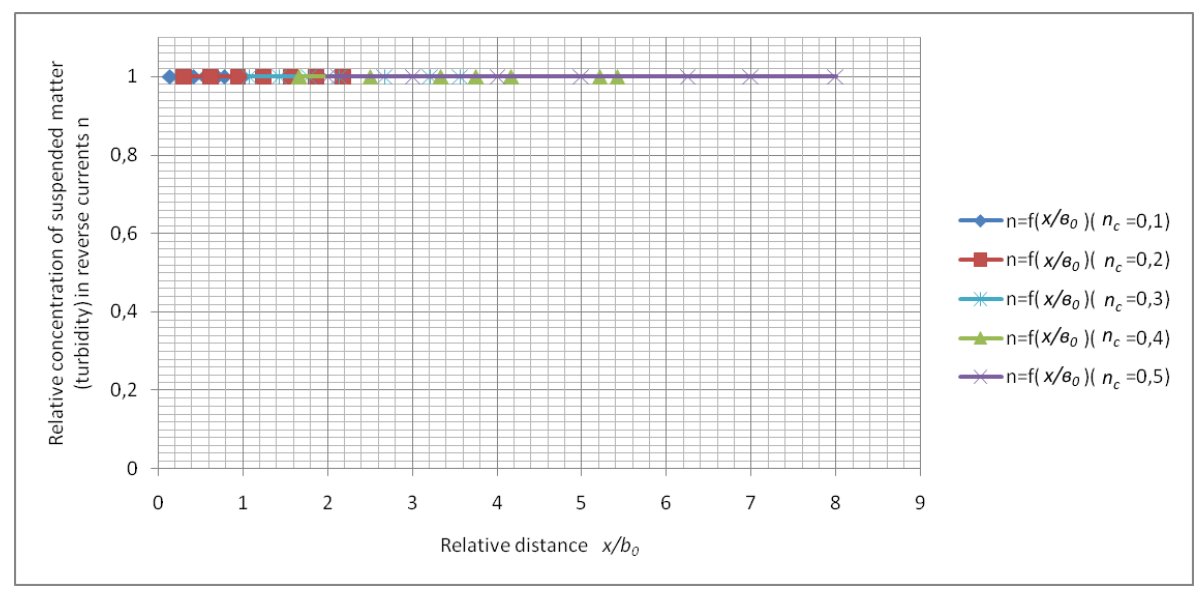

Fig. 3. Graphs of dependence of the relative concentration of suspended matter (turbidity) on the relative length of the swirling zone at various contraction ratios

As seen from the tables and graphs, the relative backflow velocities first increase and then drop sharply towards the end of the swirling zone. This tendency is observed at all contraction ratios.

The results of solving the test problem were compared (with a satisfactory agreement) with the experimental results for the case $n_{c}=0.3$ (Fig. 3).

The relative maximum backflow velocities were observed at the intersection of the outer boundary of the zone of intense turbulent mixing with the protected coast.

The relative concentration of suspended matter along the length of the swirling zone remains practically constant and is equal to unity. 


\section{Conclusions}

The theoretical dependencies obtained make it possible to calculate the velocities (11) and establish the patterns of changes in the concentration of suspended matter (12) in reverse currents.

The relative maximum velocities at all contraction ratios are 0.317 , and in the absolute value, they increase with an increase in the contraction ratio of the flow by a blind dam.

Comparing the calculated values of the backflow velocities of the test problem with the experimental values shows their qualitative and quantitative agreement.

The value of suspension concentration along the length remains constant and equal to unity.

Using the dependencies obtained (and knowing the hydraulic size of the particles), it is possible to calculate the siltation in the swirling zone in the presence of the initial section of the jet.

\section{References}

1. Decree of the Cabinet of Ministers of the Republic of Uzbekistan "On Approving the Agenda of the Republic of Uzbekistan on stabilized and safe passage of water through waterways for 2014-2015 and in perspective till 2020" N13 dated 21.01.2014.Tashkent, (2014), (in Uzbek)

2. Rakhmatov K.R. Development of floodplain lands by regulating channel deformations on the example of the Pyanj river. Dissertation. Dushanbe.(2004), dissercat.com

3. Medvedov S.S. Improvement of calculation methods for river and reclamation structures. Moscow, dissertation,(2007), dissercat.com

4. Markova I.M. Intra-channel geological processes in watercourses in urbanized areas. Moscow, dissertation, (2005), dissercat.com

5. Krilenko M.V. Study of formation mechanisms for concentration of suspended sandy sediment in riverbank zone. Dissercat http: //www.dissercat.com/content/issledovaniemekhanizmov-formirovaniya-polya-contsentratsii-vsveshennjkh-peschanikhnanosov\#ixzz580aleilm.(2007)

6. Sineeva N.V. Process of sedimentation and suspended sediment accumulation in apertures of river deltas. Yani and Indigirki. Author's abstract. Diss. c.t.s. p.27, Novosibirsk, (2006)

7. Rakhmatullaev Sh., Frederic H., Bakiev M., Mikael M-H., Philippe LeC. Sedimentation of reservoirs in Uzbekistan: a case study of the Aкdarya Reservoir, Zerafshan River Basin. HAL id:insu-00617806,https://hal-insu.archivesouvertes.fr/insu-00617806v2.

8. Vaghefi Mohammad, Safarpoor Yaser, Hashemi Seyed Shaker Civil Engineering Effects of distance between the T-shaped spur dikes on flow and scour patterns in $90^{\circ}$ bend using the SSIIM model. https://doi.org/10.1016/j.asej.2015.11.008.

9. Hossein Basser, Hojat Karami, Shahaboddin Shamshirband, Afshin Jahangirzadeh, Shatirah Akib, Hadi Saboohi Predicting optimum parameters of a protective spur dike using soft computing methodologies - A comparative study https://doi.org/10.1016/j.compfluid.2014.04.013.

10. Jennifer G. Duan, Li He, Xudong Fu, Quangqian Wang Mean flow and turbulence around experimental spur dike https://doi.org/10.1016/j.advwatres.2009.09.004.

11. Xuelin Tang, Xiang Ding, Zhicong Chen. Large Eddy Simulations of ThreeDimensional Flows Around a Spur Dike https://doi.org/10.1016/S10070214(06)70164-X. 
12. Bakiyev M.R., Improving the structure, design justification methods for regulating structures. Author's abstract of doctoral dissertation. p.57, Moscow, (1992)

13. Bakiev M.R., Togunova N.P. Hydraulic design of through-flow dikes with variable build-up. Hydraulic construction journal. №12, pp.14-17, (1989)

14. Study of submountain river flow patterns constrained by a combined dam https://iopscience.iop.org/article/10.1088/1755-1315/614/1/012053/pdf.

15. Field target dimensions of flow constrained by a transverse dam https://iopscience.iop.org/article/10.1088/1757-899X/883/1/012034/pdf.

16. Irmuxamedov X.A., Radjapov K. Design of interdam area sedimentation in Amudarya river training by transverse dams.Rep.ofUzb. ASC. Karakalpak dept. news. pp. 21-28.

17. Slautina A.V. Sedimentation of vortex zones with suspended sediment in flow spreading region. LPI works, №312, pp.20-26, (1971)

18. Distribution of turbidity in flow constrained by transverse dam https://iopscience.iop.org/article/10.1088/1757-899X/869/7/072008/pdf.

19. Abramovich G.N. Theory of turbulent streams. Fizmatgiz., p.716, Moscow, (1960)

20. Mixalev M.A. Hydraulic design of flow with vortex. p.184, Leningrad, (1971) 